\title{
MOLECULAR DOCKING OF HYRTIMOMINE A-K FROM MARINE SPONGE HYRTIOS SPP. AS ANTICANCER TARGET OF PHOSPHOINOSITIDE-DEPENDENT KINASE 1
}

\author{
RAMA ADIGA* \\ Division of Bioinformatics and Computational Genomics, Nitte University Centre for Science Education and Research, Nitte Deemed to be \\ University, Paneer Campus, Mangalore, Karnataka, India. Email: rama_adiga@nitte.edu.in
}

Received: 20 April 2019, Revised and Accepted: 15 May 2019

\begin{abstract}
Objective: The hyrtimomine A-K class of indole-based compounds extracted from Hyrtios spp. of sponges from the sea has not been studied for their anticancer properties. Phosphoinositide-dependent kinase 1 (PDK1) is a master regulator of many types of cancer. Compounds currently targeting PDK1 are currently of poor specificity and solubility. Hence, molecular docking to look for new compounds inhibiting PDK1 from the marine
\end{abstract} environment was carried out.

Methods: Target selection for ligands hyrtimomine A-K was done using PharmMapper tool. Molecular docking was done using iGEMDOCK 2.1, a generic evolutionary method of docking. Site moiety mapping was done in SimMap to extract the anchor preference of the top hits. Comparison of ligand binding energies, pharmacokinetic properties with lead compound BX-517 was carried out.

Results: Hyrtimomine B, C, D, and G were top hits using iGEMDOCK. The highest score was obtained for hyrtimomine C. Van der Waals interaction at T222 and V96 and hydrogen bond interaction at K111 determined pocket stability. The solubility properties of the compound showed higher score for hyrtimomine $\mathrm{C}$. The conserved features of hyrtimomine $\mathrm{C}$ were then compared with the crystal structure of lead compound (BX-517, which was not developed further due to poor solubility and bioavailability). The pharmacokinetic properties of hyrtimomine $\mathrm{C}$ were superior to BX-517 and had better solubility and drug-likeness score, hence, may be a candidate structure for drug development.

Conclusion: The unique azapeno indole structure of hyrtimomine $\mathrm{C}$ highlighted the mode of binding and residues in binding site.

Keywords: iGEMDOCK, Hyrtimomine, Phosphoinositide-dependent kinase 1, Cancer, Metastasis, SimMap, Hyrtios, Indole.

(C) 2019 The Authors. Published by Innovare Academic Sciences Pvt Ltd. This is an open access article under the CC BY license (http://creativecommons. org/licenses/by/4. 0/) DOI: http://dx.doi.org/10.22159/ajpcr.2019.v12i7.33673

\section{INTRODUCTION}

Phosphoinositide-dependent kinase 1 (PDK1) is a master regulator of phosphatidylinositol 3-kinase cascade and growth factor signaling that controls tumorigenesis and cancer metastasis. Proliferation of human cancers including lung, breast, gastric, ovarian, and prostate has been observed by PDK1 regulation. PDK1 belongs to AGC kinase family proteins and is a key member, involved in cAMP, cGMP, or PKC-dependent signal transduction. PDK1 has received considerable attention as a drug target in cancer therapy and as a valid target for pharmacological intervention. Inhibitors that target PDK1 provide novel opportunities and have witnessed rise of therapeutics designed toward it in recent years.

The role of PDK1 in activation of Akt has been extensively studied and the earliest mechanisms to be studied. PDK1-mediated Akt activation involves the Pleckstrin homology domain with activation of key residues by selective phosphorylation [1]. PDK1 was also found to phosphorylate other AGC kinase family members such as p70S6K, SGK, and p90RSK and the members of PKC family in a mechanism involving phosphorylation of hydrophobic motif of PIFbinding pocket and interaction by activation loop [2]. However, in cancer, metastasis overexpression of PDK1 may lead to bypassing the phosphorylation step of PIF pocket in a non-kinase-mediated direct interaction of hydrophobic motif and stabilized in activated state $[3,4]$.

Many small molecule inhibitors of PDK1 have been used on cell lines to test efficacy [5-8] including Akt inhibitors which act on cell proliferation and apoptosis [9]. Most inhibitors of PDK1 act at downstream phosphorylation sites of Akt [10-12]. Other compounds of pharmaceutical importance are naphthyridine, BX-912, etc., which show poor specificity in inhibiting PDK1 in vivo [6]. Various azaindoles have been synthesized but found to be poor in efficacy [13].

There is an urgent need for new drugs with anticancer activity. The marine environment has been reported as a rich source of natural compounds. Many compounds have been examined for in vitro and in vivo activity and pave the way for targeted therapy. About $60 \%$ of anticancer drugs available commercially are biomimetics. Anticancer activity was reported from bisindole alkaloids of marine origin (corals sponges and tunicates), particularly 2,2-bis(6-bromo-3-indolyl) ethylamine against cancer cell lines [14]. Compounds with substituted derivatives of 2-oxindole nucleus have showed apoptotic action in vitro against cancer cell lines along with cell shrinkage [15]. Oxindole derivatives have been used as anticancer agents in lung cancer [16]. Antitumor activity centered around ligands with oxindole nucleus central core are suggested to disrupt the PDK/Akt pathway. The activity of compounds is related to the presence of substituents at position 3 of the oxindole nucleus [16]. The indoles and its modifications or derived compounds possess pharmacophore activities with interesting pharmacological, antimicrobial $[17,18]$, and anticancer activities which are not yet reported. Hence, it was decided to explore the marine resources for a rare class of novel hyrtimomines from sponges for their anticancer properties and pharmacokinetic properties which would make it suitable as anticancer drug. Most drugs for cancer treatment show lack of specificity and solubility and fail at drug discovery stage. The novelty of the study is in the search for new drugs for cancer, a potential drug candidate having role as PDK1 inhibitor was used in a molecular docking approach. 


\section{METHODS}

Retrieval of small molecule inhibitors and conversion

The hyrtimomines $(\mathrm{A}-\mathrm{K})$ are a novel class of indole-based compounds. Hyrtimomine A and B have a new heteroaromatic hexacyclic fused ring system. Hyrtimomine $\mathrm{C}$, a new azepino hydroxy indole alkaloid, was discovered from a new Okinawan sponge of Hyrtios spp. [19]. Hyrtimomine $\mathrm{D}$ and $\mathrm{E}$ are structurally unique bisindole alkaloids having canthin-6-one skeleton with a hydroxyindole and an imidazolium moiety. Hyrtimomine F and G are bisindole alkaloid with the former having $\alpha$-keto- $\varepsilon$-caprolactam structure [20]. Hyrtimomine H-K is four new alkaloids with beta-carboline skeleton [21]. The structures of all the hyrtimomines given in Table 1 were downloaded from PubChem database in SDF format.

The molecules listed in Table 1 were converted to mol2 format using Avogadro software.

\section{Target prediction by PharmMapper}

The molecular file of hyrtimomines A-K downloaded from the PubChem database was uploaded into PharmMapper server. Pharmacophore mapping approach was used for potential drug target identification using PharmMapper [22]. The web server lists the top hits with annotations based on pharmacophore models in target database.

\section{Molecular docking}

Molecular docking was done using iGEMDOCK version 2.1 [23] which uses a graphical environment for virtual screening and studying pharmacological interaction for identifying lead compounds for drug target. Different stages of structure-based virtual screening are combined with post-screening analysis, a feature present in iGEMDOCK. Conserved interacting residues in binding pockets forming interaction cluster profiles are identified. Parameters of docking run in iGEMDOCK were set with population size of $n=300$, generations were set to 80 and a number of solutions were set to 10 . The best conformation with the lowest free energy of binding was selected as the best pose. A very slow docking mode was done.

\section{SimMap consensus interaction}

SimMap was used for obtaining consensus interactions between compound moieties and residues with similar physicochemical properties and to understand specificity of binding [24].

\section{Pharmacokinetic property calculator}

Osiris property explorer was employed for drug-likeness score and prediction of toxicity potential. Molinspiration tool was used to calculate the pharmacokinetic properties like Lipinski rule. Ames test for mutagenicity testing was evaluated using pre absorption, distribution, metabolism, excretion tool (ADMET). ClogP is oil/water partition coefficient, solubility and rule of five properties were calculated.

Table 1: Chemical structure of hyrtimomine A-K extracted from PubChem database and used for further docking studies

\begin{tabular}{|c|c|c|c|c|c|}
\hline No. & Compound name & Chemical structure & No. & Compound name & Chemical structure \\
\hline 1 & Hyrtimomine A & & 7 & Hyrtimomine G & \\
\hline 2 & Hyrtimomine B & & 8 & Hyrtimomine $\mathrm{H}$ & \\
\hline 3 & Hyrtimomine $\mathrm{C}$ & & 9 & Hyrtimomine I & \\
\hline 4 & Hyrtimomine D & & 10 & Hyrtimomine J & \\
\hline 5 & Hyrtimomine E & & 11 & Hyrtimomine $\mathrm{K}$ & H \\
\hline 6 & Hyrtimomine F & & & & \\
\hline
\end{tabular}




\section{RESULTS AND DISCUSSION}

The first step in drug discovery is target identification. Hence, all the molecules listed in Table 1 were subjected to PharmMapper target identification. The common targets were analyzed using Venny (http:// bioinfogp.cnb.csic.es/tools/venny/).

Ranking was carried out by fit score and Z score cutoff of 2. PDK1 crystal structure with PDBID: 2PE0 with four pharmaco features was present in all the hyrtimomines A-K. The pharmacophore model indicated that PDK1 was ideal drug target for docking with hyrtimomines A-K.

\section{Molecular docking with iGEMDOCK}

The binding site was prepared in iGEMDOCK using the PDB: 2PE0. The ligand compound in the PDB molecule 5-hydroxy-3 [(1R)-1- (1H-Pyrrol -2-yl)Ethyl]-2H-Indole-2-One was built on the lines of the lead compound BX-517 having inhibitory activity against PDK1 [25]. The crystal structure was downloaded and used as guide for docking to the hyrtimomines A-K. The interaction profile summary table scored by energy function with cutoff of -112 is listed in Table 2 . The compound included hyrtimomine $\mathrm{C}$ within the cutoff.

Generic evolutionary method of iGEMDOCK and SimMap analysis To remove the bias which energy-based scoring methods have toward polar and large molecular weight molecules, the evolutionary method of scoring was employed. The clustering of compounds and dendrogram generation of docked compounds was done based on interaction profile and atomic composition of interacting molecules in iGEMDOCK. The detailed list of compounds docked with residue numbering involved in interactions is provided in Table 2 .

The binding energy of all best-docked molecules and their interaction profile including Van der Waals (VDW): VDW forces, hydrogen bonding (H Bond) energy, electrical charge, and average configuration pair are summarized in Table 2.

The docked poses using interaction pockets for Van der Waals (labeled $\mathrm{V}$ ) and hydrogen bonding $(\mathrm{H})$ for hyrtimomine $\mathrm{C}$ are shown in Fig. 1. Hyrtimomine B, D, and G are shown in Fig. 1 (bottom panel: Leftcenter-right), respectively.

The key features (pharmacophore) are extracted using the site-moiety map and binding pocket analysis from SimMap web server. The interaction preferences of anchors and the interaction types of moiety situated in pocket are given by $\mathrm{E}$ for electrostatic interaction, $\mathrm{H}$ for hydrogen bonding, or V for VDW. The docked compounds which were top hits by SimMap analysis are shown in Table 3a. The top scoring compounds were hyrtimomine C, B, D, and G with score of $>4.20$. Hyrtimomine C Pose 4 , Pose 6, and Pose 9 were selected (Table 3a) and compared in Table 3b.

Mapping of the preferences of moieties using site-moiety map; top scoring molecules sorted on the basis of rank.

Table 2: Summary of interaction profile for best docked poses generated by iGEMDOCK scored by energy function, VDW, H bonding energy, electrical charge, and average configuration pair

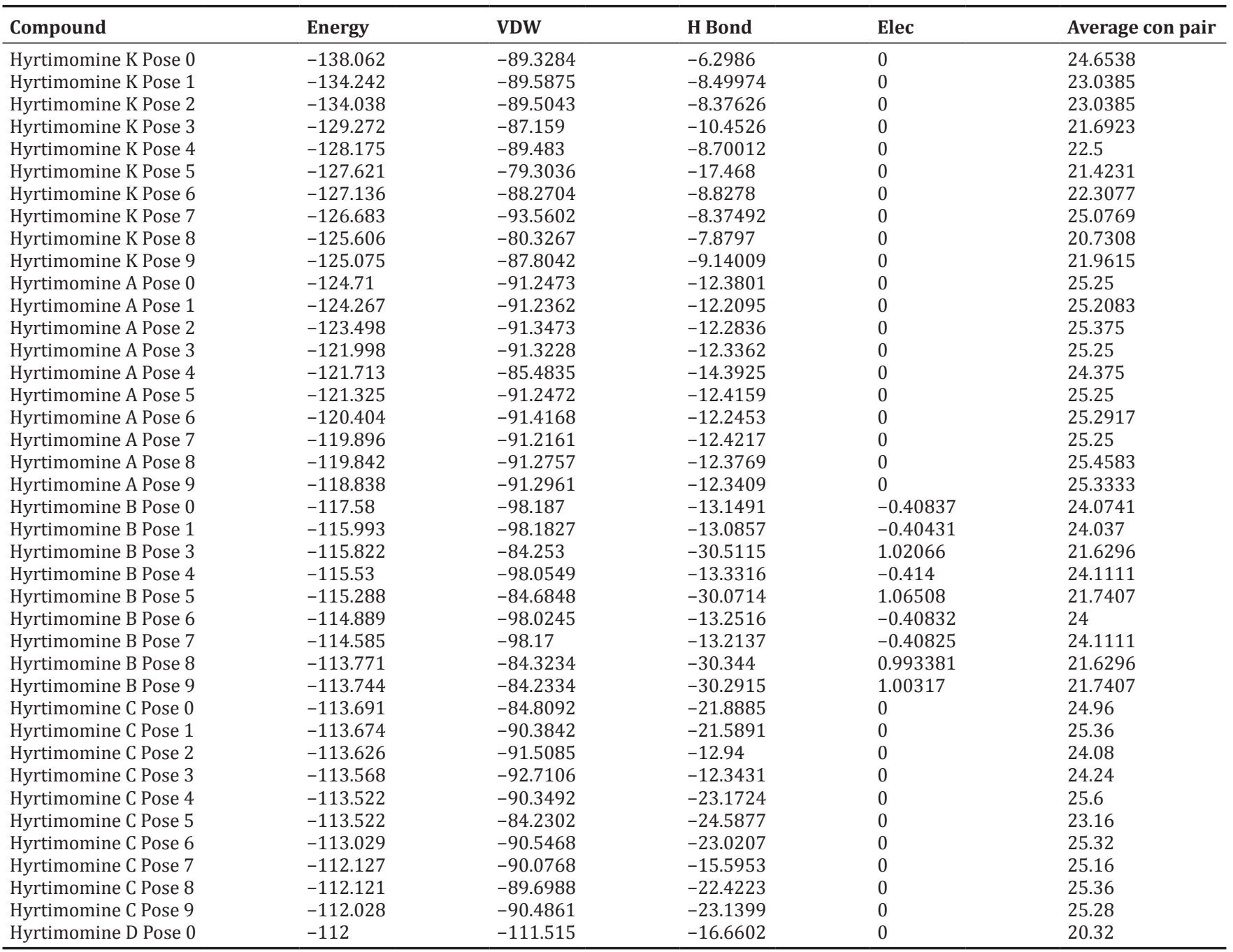

VDW: Van der Waals, H Bond: Hydrogen bonding, Elec: Electrical charge, Average con pair: Average configuration pair 
Table 3a. Comparison of hyrtimomine $\mathrm{C}$ with parameters as binding energy, VDW and $\mathrm{H}$ bond energy 3B. Comparison of lead compound BX-517 (PDK1 inhibitor) with top scoring compound hyrtimomine C.

Table 3a: Top hits by SimMap analysis

\begin{tabular}{lll}
\hline Compound docked & Rank & Score \\
\hline Hyrtimomine C Pose 4 & 1 & 4.227 \\
Hyrtimomine C Pose 6 & 2 & 4.227 \\
Hyrtimomine C Pose 9 & 3 & 4.227 \\
Hyrtimomine C Pose 1 & 4 & 4.224 \\
Hyrtimomine C Pose 8 & 5 & 4.224 \\
Hyrtimomine D Pose 8 & 6 & 4.221 \\
Hyrtimomine G Pose 9 & 7 & 4.221 \\
Hyrtimomine B Pose 1 & 8 & 4.219 \\
Hyrtimomine B Pose 3 & 9 & 4.219 \\
Hyrtimomine B Pose 5 & 10 & 4.219 \\
Hyrtimomine B Pose 8 & 11 & 4.219 \\
Hyrtimomine G Pose 7 & 12 & 4.219 \\
Hyrtimomine B Pose 9 & 13 & 4.219 \\
Hyrtimomine B Pose 0 & 14 & 4.219 \\
Hyrtimomine B Pose 2 & 15 & 4.219 \\
Hyrtimomine B Pose 4 & 16 & 4.219 \\
\hline
\end{tabular}

Table 3b: Binding energies, VDW, and $\mathrm{H}$ bonding energies of top scoring molecule hyrtimomine $C$ and lead compound BX-517 after docking with 2PE0 (PDK1) showed binding energy, VDW (Van der Waals), and H bonding similar to BX-517

\begin{tabular}{lllll}
\hline Chemical compound & Energy & VDW & H bond & Elect \\
\hline BX-517 & -103.42 & -81.39 & -22 & 0 \\
Hyrtimomine C Pose 4 & -113.52 & -90.34 & -23 & 0 \\
\hline
\end{tabular}

VDW: Van der Waals, PDK1: Phosphoinositide-dependent kinase 1, H Bond: Hydrogen bonding energy, Elec: Electrical charge
In iGEMDOCK post-run analysis procedures and SimMap analysis, the most important VDW interactions occurred with T222 and L212 (Fig. 1) while the important residues for $\mathrm{H}$ bonding were residues at $\mathrm{K} 111$ and T222. Hydrogen bonds were formed with S160 which are located in the hinge region in hyrtimomine $\mathrm{C}$.

In addition, residues in 90 loop (88-96) and S160 having interaction with main chain and side chain were found to have preference for VDW and hydrogen bond. According to SimMap analysis, there were four anchors (H1, V1-V3) with no electrostatic interaction predicted for the ligand hyrtimomine $C$. Hyrtimomine $C$ had bond length for VDW residue S94, V96, and $\mathrm{K} 111$ as $3.31 \mathrm{~A}, 3.37 \mathrm{~A}$, and $3.83 \mathrm{~A}$, respectively. Hyrtimomine B also had four anchors (H1, V1-V3) with two electrostatic interactions at K111 and D223 predicted by iGEMDOCK. Hyrtimomine $\mathrm{D}$ and $\mathrm{G}$ also had similar anchors with no electrostatic interaction. The hydrogen bond interaction was predominantly at K111 and T222.

A comparison of ligand hyrtimomine $\mathrm{C}$ and docked ligand of crystal structure is provided in Fig. 2. The novel azapeno indole structure of hyrtimomine $\mathrm{C}$ may be suitable for drug discovery.

\section{In silico pharmacokinetic property calculator}

The four compounds hyrtimomine C, B, D, and G were subjected to Osiris Property calculator for their drug-likeness and drug score. Hydrophilicity of a compound was given by its partition coefficient between log values in n-octanol and water. Poor absorption was associated with high clogP values with cutoff of 5 .

Table 4 showed the comparative values for four compounds with clogP values which was found to be $<5$. Solubility which is a factor governing poor absorption and given by $\operatorname{logS}$ values. The cutoff was set to -4 . Two compounds hyrtimomine $\mathrm{C}$ and hyrtimomine $\mathrm{G}$ had logS values $>-4$. The topological polar surface area (TPSA), a measure of the bioavailability of the drug, was found to be between 9.71 and 101.36 $\AA$ A. Hyrtimomine C, B, and D have TPSA of $110 \AA$ A. Pre ADMET was used to predict mutagenicity of compounds. Mutagenicity is tested using

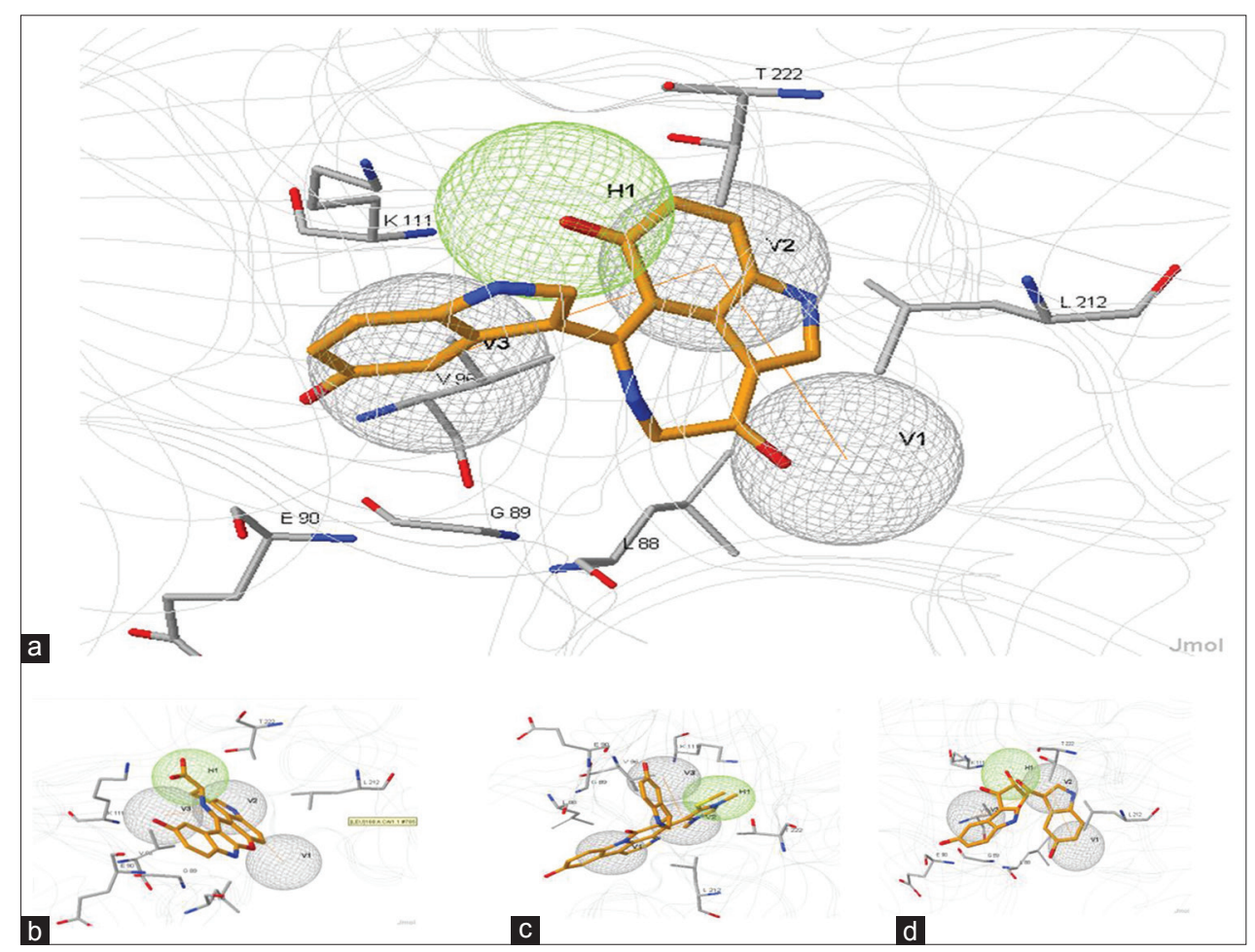

Fig. 1: (a) Top SimMap anchor preferences (H1, V1, V2, and V3) for hyrtimomine C. (b) Below left SimMap anchor preferences (H1, V1, V2, and V3) for hyrtimomine B. (c) Below middle SimMap anchor preferences (H1, V1, V2, and V3) for hyrtimomine D. (d) Below right SimMap anchor preferences (H1, V1, V2, and V3) for hyrtimomine G 
Table 4: Comparative study of hyrtimomine C, D, B, and G with BX-517 using drug toxicity and ADME properties

\begin{tabular}{|c|c|c|c|c|c|}
\hline Physicochemical properties & Hyrtimomine C & Hyrtimomine B & Hyrtimomine D & Hyrtimomine G & BX-517 \\
\hline $\operatorname{clog} \mathrm{P}$ & 1.22 & 2.71 & 0.46 & 0.34 & 0.34 \\
\hline Solubility (logS) & -3.19 & -6.46 & -6.1 & -3.82 & -2.75 \\
\hline Mol. Wt. & 331 & 359 & 508 & 380 & 282 \\
\hline TPSA & 97.98 & 110.7 & 99.44 & 146.6 & 100 \\
\hline Drug likeliness & 1.04 & -0.13 & Fail & -0.75 & 1.67 \\
\hline Drug score & 0.75 & 0.38 & Fail & 0.53 & 0.84 \\
\hline Rule of five & Suitable & Suitable & Suitable & Suitable & Suitable \\
\hline Cyp 2C Inhibitor & Non-inhibitor & Inhibitor & Inhibitor & Inhibitor & Inhibitor \\
\hline
\end{tabular}

ADME: Absorption, distribution, metabolism, and excretion

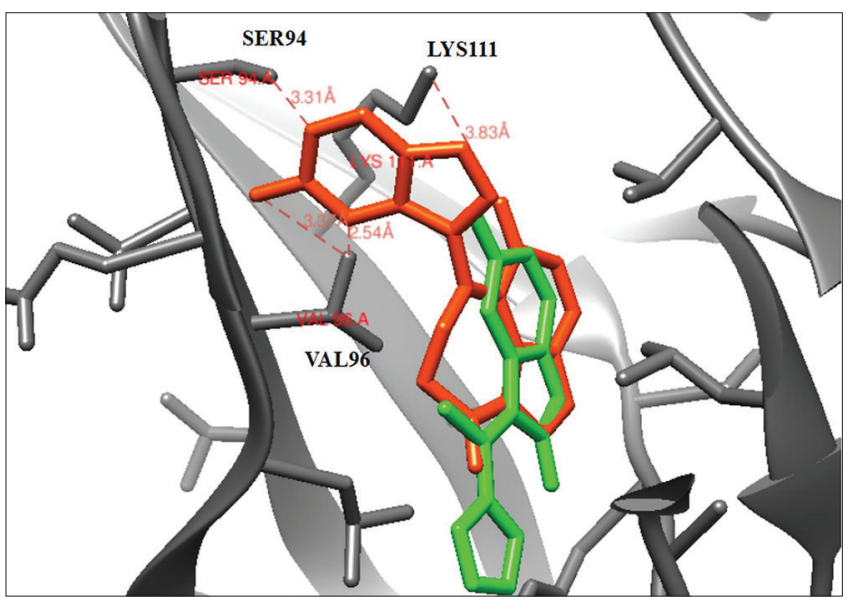

Fig. 2: Superimposed structure of hyrtimomine C (orange) with crystal structure of lead anticancer compound BX-517 (green) docked with phosphoinositide-dependent kinase 1 (PDB: 2PE0); bond length of the ligand with Van der Waals residue serine 94, valine 96, and lysine 111 is $3.31 \mathrm{~A}, 3.37 \mathrm{~A}$, and $3.83 \mathrm{~A}$, respectively.

The ligand hyrtimomine $\mathrm{C}$ (shown in orange) docked with residues in binding pocket with additional interaction from serine 94 and valine 96; BX-517 crystal structure (shown in green) had two common interacting residue lysine K111 and threonine T222

Ames test and is a major test in drug discovery. Hyrtimomine B and D do not qualify the Ames test for mutagenicity. However, hyrtimomine $\mathrm{C}$ qualifies the mutagenicity testing. The drug-likeness score was found to be highest for hyrtimomine C. An important property is inhibition of CYP2C (cytochrome P450s family) which can result in drug-to-drug interaction which is not desirable in drug discovery since it leads to delay in progress of candidate drugs.

Comparison of top scoring molecules with BX-517 (lead compound): Hyrtimomine $\mathrm{C}$ showed better solubility and clogP values than BX-517. It was predicted non-mutagenic and non-Cyp2C inhibitor indicating no drug interaction.

Only hyrtimomine $\mathrm{C}$ qualifies the CYP2C inhibition test. All the factors are listed for the four docked compounds, hyrtimomine C, B, D, and G in Table 4. Table 4 showed comparative pharmacokinetic data of the four compounds with BX-517 which is a selective PDK1 inhibitor. BX517 could not be developed into drug since it had poor solubility and the ADME properties were poor $[6,25]$. The data showed hyrtimomine $C$ had good solubility, clogP (lipophilicity) and ADME properties qualifying as the most likely candidate for drug discovery as PDK1 inhibitor. Comparative analysis of hyrtimomines and BX-517, the lead compound in cancer treatment showed the potential for the molecule to be developed into drug. In addition, the docking approach may lead to the development of new molecules which target PDK1 which has a major role in oncogenic transformation.

\section{CONCLUSION}

Drugs obtained from the marine environment have received considerable attention over the years. Hyrtimomine A-K belonging to class of indoles obtained from Hyrtios species of sponges has not been studied before and offer interesting results as anticancer targets of PDK1. Various azaindoles have been recently reviewed to find them of poor efficacy [13]. The docking results of hyrtimomine A-K with PDK1 gave top hits of four compounds hyrtimomine C, B, D, and G with score $>4.2$. The drug-likeness score and solubility factor were highest for hyrtimomine $\mathrm{C}$ which docked with PDK1. Comparative analysis of BX-517 which is a selective inhibitor of PDK1 and a lead compound showed that predicted inhibitor hyrtimomine $\mathrm{C}$ has better solubility and ADME properties than BX-517. Hence, the conserved pocket site moiety mapping and the pharmacokinetic properties of the ligand make hyrtimomine $\mathrm{C}$ valuable for drug discovery and possess the potential to be developed into drug

\section{ACKNOWLEDGMENTS}

The author wishes to thank Prof. Dr. Indrani Karunasagar, Director (Research and Development), Nitte University Centre for Science Education and Research and the Management of Nitte University, Deralakatte, Mangalore, Karnataka, India, for the support in establishing the center, providing facilities, and continuous encouragement in research, including the present work.

\section{AUTHORS' CONTRIBUTIONS}

Rama Adiga was involved in design, concept, and carrying out the docking study including writing the manuscript.

\section{CONFLICTS OF INTEREST}

There are no conflicts of interest reported and the work did not involve any financial support from any external agency.

\section{REFERENCES}

1. Milburn CC, Deak M, Kelly SM, Price NC, Alessi DR, Van Aalten DM, et al. Binding of phosphatidylinositol 3,4,5-trisphosphate to the pleckstrin homology domain of protein kinase $\mathrm{B}$ induces a conformational change. Biochem J 2003;375:531-8.

2. Biondi RM, Kieloch A, Currie RA, Deak M, Alessi DR. The PIFbinding pocket in PDK1 is essential for activation of S6K and SGK, but not PKB. EMBO J 2001;20:4380-90.

3. Leroux AE, Schulze JO, Biondi RM. AGC kinases, mechanisms of regulation and innovative drug development. Semin Cancer Biol 2018;48:1-7

4. Gagliardi PA, di Blasio L, Puliafito A, Seano G, Sessa R, Chianale F, et al. PDK1-mediated activation of $\mathrm{MRCK} \alpha$ regulates directional cell migration and lamellipodia retraction. J Cell Biol 2014;206:415-34.

5. Pinner S, Sahai E. PDK1 regulates cancer cell motility by antagonising inhibition of ROCK1 by RhoE. Nat Cell Biol 2008;10:127-37.

6. Peifer C, Alessi DR. Small-molecule inhibitors of PDK1. ChemMedChem 2008;3:1810-38.

7. Naymagon L, Abdul-Hay M. Novel agents in the treatment of multiple myeloma: A review about the future. J Hematol Oncol 2016;9:52.

8. Feldman RI, Wu JM, Polokoff MA, Kochanny MJ, Dinter H, Zhu D, 
et al. Novel small molecule inhibitors of 3-phosphoinositide-dependent kinase-1. J Biol Chem 2005;280:19867-74.

9. Keane NA, Glavey SV, Krawczyk J, O’Dwyer M. AKT as a therapeutic target in multiple myeloma. Expert Opin Ther Targets 2014;18:897 915.

10. Yang C, Huang X, Liu H, Xiao F, Wei J, You L, et al. PDK1 inhibitor GSK2334470 exerts antitumor activity in multiple myeloma and forms a novel multitargeted combination with dual mTORC1/C2 inhibitor PP242. Oncotarget 2017;8:39185-97.

11. Mora A, Komander D, van Aalten DM, Alessi DR. PDK1, the master regulator of AGC kinase signal transduction. Semin Cell Dev Biol 2004; 15:161-70.

12. Nagashima K, Shumway SD, Sathyanarayanan S, Chen AH, Dolinski B, $\mathrm{Xu}$ Y, et al. Genetic and pharmacological inhibition of PDK1 in cancer cells: Characterization of a selective allosteric kinase inhibitor. J Biol Chem 2011;286:6433-48.

13. Wucherer-Plietker M, Merkul E, Müller TJJ, Esdar C, Knöchel T, Heinrich T, et al. Discovery of novel 7-azaindoles as PDK1 inhibitors. Bioorg Med Chem Lett 2016;26:3073-80.

14. Salucci S, Burattini S, Buontempo F, Orsini E, Furiassi L, Mari M, et al. Marine bisindole alkaloid: A potential apoptotic inducer in human cancer cells. Eur J Histochem 2018;62:2881.

15. Nesi G, Sestito S, Mey V, Ricciardi S, Falasca M, Danesi R, et al. Synthesis of novel 3,5-disubstituted-2-oxindole derivatives as antitumor agents against human nonsmall cell lung cancer. ACS Med Chem Lett 2013;4:1137-41.

16. Sestito S, Nesi G, Daniele S, Martelli A, Digiacomo M, Borghini A, et al. Design and synthesis of 2-oxindole based multi-targeted inhibitors of PDK1/Akt signaling pathway for the treatment of glioblastoma multiforme. Eur J Med Chem 2015;105:274-88.

17. Jain M, Modi M, Agarwal M, Sharma H. Synthesis, free radical scavenging and DNA cleavage activities of some novel indole derivatives. Int J Pharm Pharm Sci 2015;7:97-103.

18. Rathod AS, Godipurge SS, Biradar JS. Synthesis of indole, coumarinyl and pyridinyl derivatives of isoniazid as potent antitubercular and antimicrobial agents and their molecular docking studies. Int J Pharm Pharm Sci 2017;9:233-40.

19. Momose R, Tanaka N, Fromont J, Kobayashi J. Hyrtimomines A-C, new heteroaromatic alkaloids from a sponge Hyrtios sp. Org Lett 2013;15:2010-3.

20. Tanaka N, Momose R, Nakaguchi TA, Gonoi T, Fromont J, Kobayashi J. Hyrtimomines, indole alkaloids from Okinawan marine sponges Hyrtios spp. Tetrahedron 2014;70:832-7.

21. Shady NH, Hossary EE, Fouad MA, Gulder TA, Kamel MS, Abdelmohsen UR. Bioactive natural products of marine sponges from the genus Hyrtios. Molecules 2017;22:781-802.

22. Liu X, Ouyang S, Yu B, Liu Y, Huang K, Gong J, et al. PharmMapper server: A web server for potential drug target identification using pharmacophore mapping approach. Nucleic Acids Res 2010;38:W609 14

23. Yang JM, Chen CC. GEMDOCK: A generic evolutionary method for molecular docking. Proteins 2004;55:288-304.

24. Chen YF, Hsu KC, Lin SR, Wang WC, Huang YC, Yang JM, et al. SiMMap: A web server for inferring site-moiety map to recognize interaction preferences between protein pockets and compound moieties. Nucleic Acids Res 2010;38:W424-30.

25. Islam I, Brown G, Bryant J, Hrvatin P, Kochanny MJ, Phillips GB, et al. Indolinone based phosphoinositide-dependent kinase-1 (PDK1) inhibitors. Part 2: Optimization of BX-517. Bioorg Med Chem Lett 2007; 17:3819-25. 